\title{
THE EXACT CARDINALITY OF THE SET OF TOPOLOGICAL LEFT INVARIANT MEANS ON AN AMENABLE LOCALLY COMPACT GROUP
}

\author{
ANTHONY TO-MING LAU ${ }^{1}$ AND ALAN L. T. PATERSON ${ }^{2}$
}

\begin{abstract}
The purpose of this note is to prove that if $G$ is an amenable locally compact noncompact group, then the set of topological left invariant means on $L_{\infty}(G)$ has cardinality $2^{2^{d}}$, where $d$ is the smallest cardinality of the covering of $G$ by compact sets. We also prove that in this case the spectrum of the bounded left uniformly continuous complex-valued functions contains exactly $2^{2^{d}}$ minimal closed invariant subsets (or left ideals)
\end{abstract}

1. Introduction. Let $G$ be an amenable locally compact group and $\operatorname{MTl}(G)$ be the set of all topological left invariant means on $G$. It was proved by Ching Chou in [2, Theorem 5.2] that if $G$ is a locally compact amenable group which is either $\sigma$-compact or has equivalent right and left uniform structures, then $|\operatorname{MTl}(G)|=1$ or $\geqslant 2^{c}$, where $c$ is the cardinality of the continuum. It is one if and only if $G$ is compact. (For another proof of the $\sigma$-compact case assuming the continuum hypothesis, see Granirer [6, p. 61].) He conjectured [2, p. 454] that his Theorem 5.2 is true for any locally compact group. Recently, Lau [9] showed that $|\mathrm{MTl}(G)| \geqslant 2$ if $G$ is noncompact and amenable.

Let $d(G)$ (or simply $d$ ) denote the smallest possible cardinality of a covering of $G$ by compact sets (see Liu and van Rooij [11 and 9], where the notion of $d(G)$ has been used). We prove in this paper (Theorem 1 ) that if $G$ is a noncompact locally compact amenable group, then $|\operatorname{MTl}(G)|=2^{2^{d}}$. Theorem 1 improves Theorem 5.2 of Chou [2]. It also answers affirmatively Chou's conjecture [2, p. 454]. Note that $d(G)$ is finite if and only if $G$ is compact and in this case $d(G)=1$. Also when $G$ is an infinite discrete group, then $d(G)=|G|$, and our Theorem 1 gives the well-known result of Chou [3]: $|\mathrm{MTl}(G)|=2^{2^{|G|}}$. (See also Paterson [12].)

Let $\Delta(G)$ denote the spectrum of the $C^{*}$-algebra of bounded left uniformly continuous complex-valued functions on $G$. We also prove in this paper (Proposition 2) that if $G$ is any locally compact noncompact group, then $\Delta(G)$ contains at least

Received by the editors August 16, 1985.

1980 Mathematics Subject Classification. Primary 43A07; Secondary 43A15.

Key words and phrases. Amenable locally compact groups, invariant means, left thick subsets, uniformly continuous functions, minimal invariant sets.

${ }^{1}$ Supported by NSERC Grant A-7679.

${ }^{2}$ The second author is indebted to the Royal Society and the Carnegie Trust for the Universities of Scotland for their support.

(C1986 American Mathematical Society $0002-9939 / 86 \$ 1.00+\$ .25$ per page 
$2^{2^{d}}$ minimal closed invariant subsets. Furthermore (Theorem 2), if $G$ is amenable, then the cardinality of the family of minimal closed invariant subsets of $\Delta(G)$ is exactly $2^{2^{d}}$. Proposition 2 is due to Rosenblatt [14, p. 158] (see also Remark 3 on p. 106 of [3]) when $G$ is discrete. It also improves a result of Baker and Milnes [1, p. 406].

2. Preliminaries and some notations. Throughout this paper, $G$ will denote a locally compact group with a fixed left Haar measure $\lambda$. Let $L_{\infty}(G)$ be the Banach space of essentially bounded measurable functions on $G$ with the essential sup norm $\|\cdot\|_{\infty}$ and $L_{1}(G)$ be the space of $\lambda$-integrable function $f$ on $G$ with norm $\|f\|_{1}=\int|f| d \lambda$ as defined in [8]. Let $\operatorname{LUC}(G)$ denote the space of bounded left uniformly continuous complex-valued functions on $G$, i.e. all functions of $f \in \mathrm{CB}(G)$ (bounded complex-valued continuous functions on $G$ ) such that the map $G \rightarrow \mathrm{CB}(G)$ defined by $a \rightarrow l_{a} f$ is continuous when $\operatorname{CB}(G)$ has the sup norm topology, where $\left(l_{a} f\right)(x)=f(a x), x \in G$. A positive linear functional $m$ of norm 1 on $L_{\infty}(G)$ [LUC $(G)$ ] is called a mean. A mean $m$ is called a left invariant mean if $m\left(l_{x} f\right)=$ $m(f)$ for all $x \in G, f \in L_{\infty}(G)[\operatorname{LUC}(G)]$. $G$ is amenable if $\operatorname{LUC}(G)$ has a left invariant mean. As is well known [7, Theorem 2.2.1] this is equivalent to the existence of a topological left invariant mean on $L_{\infty}(G)$, i.e. a mean $m$ such that $m(\varphi * f)=m(f), f \in L_{\infty}(G), \varphi \in L_{1}(G), \varphi \geqslant 0,\|\varphi\|_{1}=1$, where

$$
(\varphi * f)(x)=\int_{G} \varphi(y) f\left(y^{-1} x\right) d \lambda(y) .
$$

Also, there is a one-to-one correspondence between $\operatorname{Ml}(\operatorname{LUC}(G))$, the set of left invariant means on $\operatorname{LUC}(G)$ and $\operatorname{MTl}(G)$ given by the restriction map (see [2, Lemma 2.2]).

Let $\Delta(G)$ denote the spectrum of the commutative $C^{*}$-algebra $\operatorname{LUC}(G)$, i.e. the set of nonzero multiplicative linear functionals on $\operatorname{LUC}(G)$ with the relative weak*topology. Then there is a natural jointly continuous action of $G$ on $\Delta(G)$ defined by the map $(g, \varphi) \rightarrow l_{g}^{*} \varphi$ where $\left\langle l_{g}^{*} \varphi, f\right\rangle=\varphi\left(l_{g} f\right), g \in G, \varphi \in \Delta$ and $f \in \operatorname{LUC}(G)$. A subset $C$ of $\Delta(G)$ is invariant if $l_{g}^{*}(C) \subseteq C$ for each $g \in G$. Furthermore, a closed subset $C$ of $\Delta(G)$ is invariant if and only if $C$ is a left ideal in $\Delta(G)$ with multiplication $\odot$ defined by $\langle m \odot n, f\rangle=\left\langle m, n_{l}(f)\right\rangle, m, n \in \Delta(G), f \in \operatorname{LUC}(G)$, where $n_{l}(f)(g)=\left\langle n, l_{g} f\right\rangle, g \in G$. Minimal left ideals in $\Delta(S)$ must clearly be closed.

3. Topologically disjoint left thick subsets in a locally compact group. Let $\alpha=\alpha(G)$ be the smallest ordinal having cardinality $d(G)$. Let $\left\{K_{\beta} ; 1 \leqslant \beta<\alpha\right\}$ be a family of compact subsets of $G$ covering $G$. We may assume, without loss of generality, that $\left\{K_{\beta} ; 1 \leqslant \beta<\alpha\right\}$ is closed under finite unions.

LEMMA 1. Let $U$ be a compact neighborhood of the identity e of $G$. Then there exists a subset $\left\{x_{\beta \gamma}: 1 \leqslant \beta \leqslant \gamma<\alpha\right\}$ of $G$ such that the family $\left\{U K_{\gamma} x_{\beta \gamma} ; 1 \leqslant \beta \leqslant \gamma<\alpha\right\}$ is pairwise disjoint. 
PRoof. If we agree that $\left(\gamma_{1}, \beta_{1}\right)<\left(\gamma_{2}, \beta_{2}\right)$ whenever $\gamma_{1}<\gamma_{2}$ or whenever $\gamma_{1}=\gamma_{2}$ and $\beta_{1}<\beta_{2}$, then $\{(\gamma, \beta) ; 1 \leqslant \beta \leqslant \gamma<\alpha\}$ is a well-ordered set. We shall define the sequence $\left\{x_{\beta \gamma}: 1 \leqslant \beta \leqslant \gamma<\alpha\right\}$ by transfinite induction. Let $x_{11}$ be an arbitrary element of $G$. Suppose that $x_{\beta \gamma}$ has already been defined for all pairs $(\gamma, \beta)<$ $\left(\gamma_{0}, \beta_{0}\right), 1 \leqslant \beta \leqslant \gamma$. Consider the union $W\left(\gamma_{0}, \beta_{0}\right)$ of the sets $U K_{\gamma} x_{\beta \gamma}$ as $(\gamma, \beta)$ runs over all pairs $(\gamma, \beta)<\left(\gamma_{0}, \beta_{0}\right)$. Then $K_{\gamma_{0}}^{-1} U^{-1} W\left(\gamma_{0}, \beta_{0}\right)$ admits a compact covering of cardinality less than $d(G)$ and hence cannot be all of $G$. Now choose $x_{\beta_{0} \gamma_{0}}$ to be any element of $G \sim K_{\gamma_{0}}^{-1} U^{-1} W\left(\gamma_{0}, \beta_{0}\right)$ to complete the inductive argument.

A subset $F$ of $G$ is left thick if for any finite subset $\sigma$ of $G$ there exists $a \in G$ such that $\sigma a \subseteq F$ (see Mitchell [10]). A family $\left\{Z_{\beta} ; \beta \in N\right\}$ of left thick subsets of $G$ is said to be topologically disjoint if

(i) For any subset $N_{0}$ of $N$, there exists a function $\varphi \in \operatorname{LUC}(G)$ such that $\varphi(x)=1$ for all $x \in Z_{\beta}, \beta \in N_{0}$, and $\varphi(x)=0$ for all $x \in Z_{\beta}, \beta \notin N_{0}$.

The following proposition is the key to the proof of our main results.

Proposition 1. Let $G$ be a locally compact group. There exists a topologically disjoint family of left thick subsets $\left\{Z_{\beta} ; \beta \in N\right\}$ of $G$ such that $|N|=d$.

Proof. Let $U$ be a compact neighbourhood of $e$. Let $\left\{x_{\beta \gamma}: 1 \leqslant \beta \leqslant \gamma<\alpha\right\}$ be as in Lemma 1. Let $N=\{\beta ; \beta$ is an ordinal and $1 \leqslant \beta<\alpha\}$. For each $\beta \in N$, let $Z_{\beta}=\bigcup\left\{K_{\gamma} x_{\beta \gamma} ; 1 \leqslant \beta \leqslant \gamma<\alpha\right\}$. Clearly $|N|=d$. Also each $Z_{\beta}$ is left thick. Indeed, if $\sigma$ is a finite subset of $G$, then $\sigma \subseteq K_{\gamma}$ for some $\gamma<\alpha$. So $\sigma x_{\beta \gamma} \subset Z_{\beta}$.

To see that the family $\left\{Z_{\beta} ; \beta \in N\right\}$ satisfies (i), let $V$ be a compact symmetric neighbourhood of $e$ such that $V^{3} \subseteq U$. Using Urysohn's lemma, we can find a continuous function $f: G \rightarrow[0,1]$ such that $f(e)=1$ and $f(G \sim V)=\{0\}$. Following an idea as in the proof of Theorem 1 [9], define a pseudometric $d$ of $G$ by setting

$$
d(x, y)=\left\|l_{x} f-l_{y} f\right\| \quad(x, y \in G),
$$

and for $1 \leqslant \beta \leqslant \gamma<\alpha$, define a function $g_{\beta \gamma}$ on $G$ by setting

$$
g_{\beta \gamma}(x)=1-d\left(x, K_{\beta \gamma}\right) \text {, }
$$

where $x \in G$ and $K_{\beta \gamma}=K_{\gamma} x_{\beta \gamma}$. Clearly, $g_{\beta \gamma}$ is continuous, $0 \leqslant g_{\beta \gamma} \leqslant 1$, and $g_{\beta \gamma}(x)=1$ for all $x \in K_{\beta \gamma}$. Furthermore, if $g_{\beta \gamma}(x)>0$, then $x \in V^{2} K_{\beta \gamma}$. (Indeed, in that case, $d(x, y)<1$ for some $y \in K_{\beta \gamma}$, and hence $V x \cap V y \neq \varnothing$. For otherwise $\left(l_{x} f\right)\left(x^{-1}\right)=1$ and $\left(l_{y} f\right)\left(x^{-1}\right)=0$ and $d(x, y)=1$.)

Define, for each $\beta \in N, \varphi_{\beta}=\sum\left\{g_{\beta \gamma} ; \beta \leqslant \gamma<\alpha\right\}$. Since the family $\left\{U K_{\beta \gamma}\right.$; $1 \leqslant \beta \leqslant \gamma<\alpha\}$ is pairwise disjoint, it follows that $\varphi_{\beta}$ is well defined and $0 \leqslant \varphi_{\beta}(x)$ $\leqslant 1$ for each $x \in G$. Also, each $\varphi_{\beta}$ is left uniformly continuous. Indeed, let $x \in V$ and suppose that $t \in G$ such that $\left|\varphi_{\beta}(x t)-\varphi_{\beta}(t)\right|>0$. If $\varphi_{b}(x t) \neq 0$, then $x t \in$ $V^{2} K_{\beta \gamma}$ for some unique $\gamma$, and this gives $t \in V^{3} K_{\beta \gamma} \subseteq U K_{\beta \gamma}$. Similarly, if $\varphi_{\beta}(t) \neq 0$, then both $x t$ and $t$ are in $U K_{\beta \gamma}$ for unique $\gamma$. Thus

$$
\begin{aligned}
\left|\varphi_{\beta}(x t)-\varphi_{\beta}(t)\right| & =\left|g_{\beta \gamma}(x t)-g_{\beta \gamma}(t)\right|=\left|d\left(t, K_{\beta \gamma}\right)-d\left(x t, K_{\beta \gamma}\right)\right| \\
& \leqslant d(t, x t)=\left\|l_{x} f-f\right\| .
\end{aligned}
$$

Consequently $\left\|l_{x} \varphi_{\beta}-\varphi_{\beta}\right\| \leqslant\left\|l_{x} f-f\right\|$. Hence $\varphi_{\beta} \in \operatorname{LUC}(G)$ since $f \in \operatorname{LUC}(G)$.

Finally if $N_{0}$ is a subset of $N$, let $\varphi=\sum\left\{\varphi_{\beta} ; \beta \in N_{0}\right\}$. Then $\varphi$ satisfies (i). 
4. Minimal closed invariant subsets of $\Delta(G)$. If $g \in G$, let $\delta_{g} \in \Delta(G)$ be defined by $\delta_{g}(f)=f(g), f \in \mathrm{LUC}(g)$. For any subset $F \subseteq G$, let $F^{-}$denote the closure of $\left\{\delta_{g} ; g \in F\right\}$ in $\Delta(G)$.

LEMma 2. Let $\left\{F_{u} ; u \in \Gamma\right\}$ be a collection of subsets of $G$ such that for any $u_{1}, \ldots, u_{n} \in \Gamma, \cap\left\{F_{u_{i}} ; i=1, \ldots, n\right\}$ is left thick. Then the set $C=\cap\left\{\left(g F_{u}\right)^{-} ; g \in G\right.$, $u \in \Gamma\}$ is nonempty, compact, and invariant in $\Delta(G)$.

Proof. For any finite subset $\sigma$ of $G$ and $u_{1}, \ldots, u_{n} \in \Gamma, \cap\left\{g F_{u_{i}} ; g \in \sigma, i=\right.$ $1, \ldots, n\}$ is nonempty by left thickness of $\cap\left\{F_{u_{i}} ; i=1, \ldots, n\right\}$. Hence $C$ is nonempty and compact. The invariance of $C$ is immediate.

LEMMA 3. Let $G$ be a noncompact locally compact group. If $G$ contains a topologically disjoint infinite family $\left\{Z_{\beta} ; \beta \in N\right\}$ of left thick subsets, then $\Delta(G)$ contains at least $2^{2^{|N|}}$ minimal closed invariant subsets.

Proof. By Lemma 2 in [3], we can find a family $\left\{N_{u} ; u \in \Gamma\right\}$ of subsets of $N$ such that $|\Gamma|=2^{d}$ and whenever $u_{1}, \ldots, u_{n}$ are distinct elements of $\Gamma$ and $\varepsilon_{i} \in\{1, c\}$, where, for $A \subset N, A^{1}=A, A^{c}=N \sim A$, then $\bigcap_{i=1}^{m} N_{u_{i}}^{\varepsilon_{i}} \neq \varnothing$. Let $P=\{1, c\}^{\Gamma}$ so that $|P|=2^{2^{d}}$. For $u \in \Gamma$, let $E_{u}=\bigcup\left\{Z_{\beta} ; \beta \in N_{u}\right\}$. For $p \in P$, let

$$
C_{p}=\bigcap\left\{\left(g E_{u}^{p(u)}\right)^{-} ; g \in G, u \in \Gamma\right\}
$$

(here $E_{u}^{1}=E_{u}, E_{u}^{c}=\left[\bigcup\left\{K_{\gamma} x_{\beta \gamma} ; 1 \leqslant \beta \leqslant \gamma<\alpha\right\}\right] \sim E_{u}$ ). By Lemma 2, each $C_{p}$ is nonempty, compact, and invariant (hence must contain a nonempty minimal closed invariant subset). We now show that

$$
C_{p} \cap C_{q}=\varnothing \quad \text { for } p \neq q \text {. }
$$

Indeed, if $p \neq q$, there exists $u_{0} \in \Gamma$ such that $p\left(u_{0}\right) \neq q\left(u_{0}\right)$. Suppose, without loss of generality, that $p\left(\mathrm{u}_{0}\right)=1, q\left(u_{0}\right)=c$. Then

$$
C_{p} \subset\left(E_{u_{0}}\right)^{-}, \quad C_{q} \subset\left(E_{u_{0}}^{c}\right)^{-} .
$$

By (i), there exists $\varphi \in \operatorname{LUC}(G)$ such that $\varphi(x)=1$ for all $x \in Z_{\beta}, \beta \in N_{u_{0}}$, and $\varphi(x)=0$ for all $x \in Z_{\beta}, \beta \in N_{u_{0}}$. In particular, $C_{p} \cap C_{q}=\varnothing$.

Baker and Milnes [1, Remark 2, p. 406] prove that if $G$ is any noncompact abelian group, then $\Delta(G)$ contains at least $2^{c}$ minimal closed invariant subsets (left ideals). The following is an improvement of their result (see Theorem 2).

Proposition 2. Let $G$ be a noncompact locally compact group. Then $\Delta(G)$ contains at least $2^{2^{d}}$ minimal closed invariant subsets.

Proof. Use Proposition 1 and Lemma 3.

COROLlaRY. Let $G$ be a locally compact group. Then $\Delta(G)$ has finitely many minimal closed invariant subsets if and only if $G$ is compact.

\section{The main results.}

LEMMA 4. Let $G$ be an amenable locally compact group. If $\Delta(G)$ contains $n$ minimal closed invariant subsets, then $|\mathrm{MTl}(G)| \geqslant n$. 
Proof. Let $C$ be a closed invariant subset of $\Delta(G)$. Let $M$ be the weak*-closed convex hull of $C$ in $\operatorname{LUC}(G)^{*}$. Then $M$ is also $G$-invariant and the action of $G$ on $M$ defined by $(g, m) \rightarrow l_{g}^{*} m$ is jointly continuous. Hence by Day's fixed point theorem [4 and 5], there exists $m_{0} \in M$ such that $l_{g}^{*} m_{0}=m_{0}$ for all $g \in G$. Furthermore, the support of $m_{0}$ (regarded as a regular Borel measure on $\Delta$ ) is contained in $C$. Hence

$$
|\operatorname{MTl}(G)|=|\operatorname{Ml}(\operatorname{LUC}(G))| \geqslant n .
$$

THEOREM 1. Let $G$ be a noncompact amenable locally compact group. Then $|\operatorname{MTl}(G)|=2^{2^{d}}$.

Proof. By Proposition 2 and Lemma 4, $|\operatorname{MTl}(G)| \geqslant 2^{2^{d}}$. To prove the converse, let $H$ be a $\sigma$-compact noncompact open subgroup of $G$ (see [2, Lemma 4.8]). We can find a compact normal subgroup $K$ of $H$ such that $H / K$ is separable (see [8, p. 71]). Let $\mu$ be the normalized Haar measure on $K$ regarded as a probability measure in $M(G)$. Let $\nu \in L_{1}(G), \nu \geqslant 0$ and $\|\nu\|_{1}=1$. Let $\theta=\mu * \nu$. Then $\theta \in L_{1}(G)$, $\|\theta\|_{1}=1$, and $\theta_{1} \geqslant 0$. Also $\langle m, \theta * f\rangle=\langle m, f\rangle$ for each $f \in L_{\infty}(G)$ and $m \in$ $\operatorname{MTl}(G)$. Consequently, each $m \in \operatorname{MTl}(G)$ may be regarded as a continuous linear function on the subspace $A=\left\{\theta * f ; f \in L_{\infty}(G)\right\}$ contained in $\operatorname{LUC}(G)$. Furthermore, each function in $A$ is constant on right cosets of $G$ with respect to the subgroup $K$. Consequently, $A$ may be regarded as a subspace of $\mathrm{CB}(G \backslash K)$, where $G \backslash K$ denotes the right coset space of $G$ with respect to $K$. Clearly, the smallest possible covering of $G \backslash K$ by compact sets has cardinality $d$. Also, every compact subset $C$ of $G \backslash K$ is separable (since $C$ can be covered by finitely many open sets of the form $(H \backslash K) \cdot g, g \in G$, and each $(H \backslash K) \cdot g$ is separable). Hence we can find a dense subset $T$ of $G \backslash K$ of cardinality $\boldsymbol{\aleph}_{0} \cdot d=d$. Since every function in $\mathrm{CB}(G \backslash K)$ is determined by its value on $T$, we have $|\mathrm{CB}(G \backslash K)| \leqslant c^{d}=2^{d}$. So

$$
|\operatorname{MTl}(G)| \leqslant\left|A^{*}\right| \leqslant\left|\mathrm{CB}(G \backslash K)^{*}\right| \leqslant c^{2^{d}}=2^{2^{d}} .
$$

This completes the proof of Theorem 1 .

REMARK. It follows from Theorem 1 that if $G$ is a noncompact amenable locally compact group, then the dimension of the linear span of the set of topological left invariant means is infinite. Also, in this case, the dimension of the radical of the Banach algebra $\operatorname{LUC}(G)^{*}$ is at least $2^{2^{d}}$. (See discussion after Theorems 5.2 and 5.5 in Chou [2] and Chapter III in Granirer [6].)

THEOREM 2. Let $G$ be a noncompact amenable locally compact group. Then $\Delta(G)$ contains exactly $2^{2^{d}}$ minimal closed invariant subsets.

Proof. This follows from Lemma 4 and Proposition 2.

\section{REFERENCES}

1. J. W. Baker and P. Milnes, The ideal structure of the Stone-Čech compactification of a group, Math. Proc. Cambridge Philos. Soc. 82 (1977), 401-409.

2. C. Chou, On topological invariant meas on a locally compact group, Trans. Amer. Math. Soc. 151 (1970), 443-456.

3. The exact cardinality of the set of invariant means on a group, Proc. Amer. Math. Soc. 55 (1976), 103-106. 
4. M. M. Day, Fixed-point theorems for compact convex sets, Illinois J. Math. 5 (1961), 585-590.

5. __ Correction to my paper "Fixed-point theorems for compact convex sets", Illinois J. Math. 8 (1964), 713.

6. E. E. Granirer, Exposed points of convex sets and weak sequential convergence, Mem. Amer. Math. Soc. No. 123(1972).

7. F. P. Greenleaf, Invariant means on topological groups, Van Nostrand Math. Studies, Van Nostrand Reinhold, 1969.

8. E. Hewitt and K. Ross, Abstract harmonic analysis 1, Springer-Verlag, Berlin and New York, 1963.

9. A. T. Lau, Continuity of Arens multiplication on the dual space of bounded uniformly continuous functions on locally compact groups and topological semigroups, Math. Proc. Cambridge Philos. Soc. (to appear).

10. T. Mitchell, Constant functions and left invariant means on semigroups, Trans. Amer. Math. Soc. 119 (1965), 244-261.

11. T. S. Liu and A. van Rooij, Invariant means on a locally compact group, Monatsh. Math. 78 (1974), 356-359.

12. A. L. T. Paterson, The cardinality of the set of left invariant means on a left amenable semigroup, Illinois J. Math. 29 (1985), 567-583.

13. J. P. Pier, Amenable locally compact groups, Wiley, New York, 1984.

14. J. M. Rosenblatt, The number of extensions of an invariant mean, Compositio Math. 33 (1976), 147-159.

Department of Mathematics, University of Alberta, Edmonton, Alberta, Canada T6G 2G1

Department of Mathematics, University of Aberdeen, Aberdeen AB9 2TY, Scotland, United KINGDOM 